\title{
Controlling Epidemic Diseases Based only on Social Distancing Level
}

\author{
Samaherni Dias $^{1}\left[\right.$ [D $\cdot$ Kurios Queiroz ${ }^{1} \cdot$ Aldayr Araujo $^{1}$ \\ Received: 20 November 2020 / Revised: 14 May 2021 / Accepted: 30 May 2021 / Published online: 8 June 2021 \\ (c) Brazilian Society for Automatics-SBA 2021
}

\begin{abstract}
On March 11, 2020, the world health organization (WHO) characterized COVID-19 as a pandemic. When the COVID19 outbreak began to spread, there was no vaccination and no treatment. To epidemic diseases without vaccines or other pharmaceutical intervention, the only way to control them is a sustained physical distancing. In this work, we propose a simple control law to keep the epidemic outbreak controlled. A sustained physical distancing level is adjusted to guarantee the fastest way to finish the outbreak with the number of hospitalized individuals below the desired value. This technique can reduce the economic problems of social distancing and keeps the health care system working. The proposed controller is a closed-loop approach that uses the number of hospitalized individuals as the feedback signal. It also does not need massive swab tests, which simplify the application of the technique. We do stability analyses of the proposed controller to prove the robustness to uncertainties in the parameters and unmodeled dynamics. We present a version of the proposed controller to operate using steps to reopen, which is relevant to help the decision-makers. The proposed controller is so simple that we can use a spreadsheet to calculate the physical distancing level. In the end, we present a set of numerical simulations to highlight the behavior of the number of hospitalized individuals during an epidemic disease when using the proposed control law. We simulate the proposed controller applied to the ideal case, considering uncertainties, unmodeled dynamics, a 10 days latent period, and different values of the desired number of hospitalized individuals. In all cases, the proposed controller ensures the number of hospitalized individuals lower than the upper limit of a predefined range.
\end{abstract}

Keywords COVID-19 · Epidemic disease · SIR model · Controller

\section{Introduction}

As of March 11, 2020, the world health organization (WHO) assessed COVID-19 as a pandemic. This assessment changed the researchers' attention to the novel severe acute respiratory syndrome coronavirus 2 (SARS-CoV-2).

In a realistic scenario of an epidemic disease, the available social and medical resources to treat diseases or prevent their spreading are usually limited (Jiang and Zhou 2018). The COVID-19 pandemic has the basic reproduction number relatively high and presents worrying hospitalization and death rates (Verity et al. 2020).

Measures used to prevent or control infectious diseases are vaccination, treatment, quarantine, isolation, and prophylaxis. Quarantine and isolation are two measures by which

\section{Samaherni Dias}

sama@laci.ufrn.br

1 Laboratory of Automation, Control, and Instrumentation Department of Electrical Engineering, Federal University of Rio Grande do Norte, Natal, RN, Brazil exposed or infectious individuals are removed from the population to prevent the spread of infection. They are applied less often. Quarantine is one of the first response methods used in an extreme emergency. Quarantine was implemented during the SARS epidemic of 2002-2003 (Martcheva 2015).

In the literature, we find some control schemes for epidemic diseases. Some of them use modern theories and other traditional theories. For example, we can highlight works on the optimal control theory (Martcheva 2015; Ball et al. 2008; Gaff and Schaefer 2009; Zaman et al. 2009). The optimal control theory also was applied for the COVID-19 outbreak (Tsay et al. 2020; Köhler et al. 2020; Perkins and España 2020; Kantner and Koprucki 2020; Borri et al. 2020). An investigation of adaptive strategies to control the COVID19 is the result of (Köhler et al. 2020) work. Perkins and España (2020) determines optimal control strategies for nonpharmaceutical interventions in COVID-19. Kantner and Koprucki (2020) uses Perkins' similar approach. Tsay et al. (2020) shows a dynamic optimization-based approach to the entire epidemiological modeling and outbreak control work- 
flow. The focus of Borri et al. (2020) was lock-down and reopening policies to minimize the COVID-19 impacts.

Additionally to optimal control, we have the Model Predictive Controllers. Sélley et al. (2015) applied a nonlinear model predictive control to model a susceptible-infectioussusceptible epidemic on nontrivial contact structures. To capture the key dynamical properties of COVID-19, Péni et al. (2020) proposes a model predictive approach for constrained control. Morato et al. (2020a) formulates a Model Predictive Control policy to mitigate the COVID-19 contagion in Brazil. In this last work, to reduce the COVID-19 effects, Morato et al. (2020b) proposes a Nonlinear Model Predictive Control scheme to plan appropriate social distancing measures.

Another approach to control epidemic diseases is the feedback controller theory (Stewart et al. 2020; Di Lauro et al. 2021; Casella 2021). Stewart et al. (2020) analyzed some scenarios of commonly used infectious-disease computer models for different levels of feedback. In Di Lauro et al. (2021), the authors investigate the use of feedback control theory as a tool for engineering an effective curve flattening strategy. Casella (2021) studies if and to which extent COVID-19 epidemics can be controlled by authorities taking decisions on public health measures based on daily reports of swab test results, active cases, and total cases. According to Casella (2021), the analysis shows that suppression strategies can be effective if strong enough and enacted early on.

A challenge to optimization concepts is to obtain accurate estimates of key model parameters (Tsay et al. 2020). This question is also pointed by Perkins and España (2020), which emphasizes the importance of "careful estimation" of the model parameters. However, to Gaff and Schaefer (2009), the optimal control theory is a valuable tool to help in the adjustment of trade-offs between vaccination and treatment strategies. As optimal control, the model predictive control needs a careful estimation to achieve accurate estimates of model parameters. The epidemic models are nonlinear systems, and the design of a state estimator for these systems is non-trivial (Isidori 1995; Casella 2021) claims the feedback control theory is a powerful tool to support the management of the COVID-19 outbreak. Most advanced feedback control methods need all state information for computing the input, which is not realistic to assume (Péni et al. 2020). This assumption implies that the number of individuals in each compartment is continuously measured.

According to Köhler et al. (2020), social distancing is an effective way to contain the spread of a contagious disease, especially when we unknow the virus and no vaccines or other pharmaceutical interventions are available. To Prem et al. (2020), the sustained physical distancing can reduce the magnitude of the epidemic peak of COVID-19 and lead to a smaller number of overall cases. In the work of Hellewell et al. (2020), the authors conclude that in most scenarios, highly effective contact tracing and case isolation is enough to control a new outbreak of COVID-19.

In literature, several control schemes use deterministic compartmental models. They are compartmental because they divide the population into compartments. Each individual can reside in exactly one compartment, and it can move between compartments (Martcheva 2015). Deterministic compartmental models require well-mixed populations, which is rare in real epidemic diseases. Besides that, these models are inadequate to represent all effects of epidemic disease. For example, crowding phenomenon, multi-strains, time-varying infectivity, superinfection, etc. Thus, using these models to describe an epidemic disease during the outbreak is not adequate. To use them as real-time models can be dangerous because they have uncertainties in the parameters and suffer from unmodeled dynamics. The model errors during an epidemic outbreak can compromise the strongly model-dependent control laws.

It is necessary to understand the biological aspects of epidemic disease to propose a viable control law. The spread of an infectious disease shows a duality. It is a random process in a small group of individuals and, when the group of individuals is very large, it shows deterministic characteristics (Daley and Gani 1999). Add to this duality the variabilities associated with individual dynamics and with the environment (Allen 2017). Another element that is crucial to all such models is network topology lack (Newman 2002). Deterministic epidemic models have several challenges (Roberts et al. 2015), and this way, the control scheme based on it has challenges too. We highlight two of these challenges: uncertainties in the parameters (Nowzari et al. 2016) and stochastic effects (Roberts et al. 2015). From the Ker model until nowadays, the researchers are presenting new models to achieve better results. The better models are so complex which impose complex control schemes.

There are several control law propositions, and each one of them has qualities and challenges. Complex control schemes keep decision-makers away from them. ${ }^{1}$ We can find in the literature some works showing excellent results applying different control schemes to epidemic diseases. However, the reader must comprehend that the results are to the models used to simulate the epidemic disease, which implies the reader has to analyze the model to a real-time situation and the impacts in the control law. The wrong model, to model-dependent controllers, implies wrong control actions resulting in a chain of errors that can be amplified very fast.

During the COVID-19 outbreak, new challenges emerged: the health care system collapse and the economic crisis. To epidemic diseases without treatment and vaccines, it is nec-

\footnotetext{
${ }^{1}$ In most control system applications, $90-95 \%$ of control loops are of PID form due to their simple structure, easy implementation, and maintenance (Borase et al. 2021).
} 
essary to define an accurate social distancing level to avoid tragic effects on the population due to economic or health aspects. According to Morato et al. (2020a), countries that implement rigid-social distancing measures have seen devastating economic effects. To Borri et al. (2020), the diffusion of COVID-19 represents a real threat to the country's health and economic system. On the other hand, it is necessary to maximize physical distancing to avoid the collapse of the healthcare system. An epidemic disease without control shows exponential growth, which implies a large number of infected individuals, at the same time, requiring the health care system.

Some countries have no savings to support a long period of social distancing or even high-level distancing measures for a short time. Generally, these same countries have a low level of hospital beds. These countries need a control law to calculate the adequate social distancing level. In other words, to define the minimum social distancing level to avoid the collapse of the healthcare system.

Contributions This work proposes a feedback controller with low model dependency to control epidemic diseases that do not have vaccination and treatment. The aim is to design a local level simple rule to adjust a trade-off between the health care and the socio-economic cost of the pandemic. The proposed controller is robust to uncertainties in the parameters and unmodeled dynamics, is based on daily reports, and only uses social distancing level to act in the epidemic outbreak. The daily reports considered here are the measurements of hospitalized individuals due to the epidemic disease. We consider the social distancing level as the number of individuals, infected or not, removed from the population to prevent the spread of infection.

The paper is organized as follows. We introduce the epidemic SIR model in Sect. 2. In Sect. 3, we present a controller, considering the uncertainties of the process, to a classical model of the epidemic disease. Section 4 contains some numerical simulations, and Sect. 5 provides some concluding comments.

\section{SIR Model}

Kantner and Koprucki (2020) claims that mathematical modeling of the spread of epidemics is an indispensable tool to project a control law to an epidemic outbreak, estimate important epidemiological parameters, and make predictions for different scenarios. However, defining the correct model is a complex activity. According to Daley and Gani (1999), the spread of an infectious disease is a random process in a small group of individuals. When the number of individuals is enormous, it is customary to represent the infection process deterministically. In addition to the Daley and Kantner claims, Zhang et al. (2015) highlights that social contact networks have a community structure. To them, the population is divided into clusters with different dynamics to infected individuals.

In this work, we pretend to design a local level simple rule to the control law. In this way, we ignore all complexity involved in the spread of an infectious disease. We will work only with the fundamental dynamics of the process that is relevant for feedback control. Thus, assuming that the population is sufficiently well mixed, we will describe the dynamic of epidemic diseases by the Susceptible-InfectiousRecovered (SIR) model.

Kermack et al. (1927) formulated the SIR model. The SIR model applies to epidemics having a relatively short duration (few months). The model presented in this work is to epidemic diseases with no treatment and vaccination. Consider a SIR-type model consisting of a system of three differential equations:

$$
\left\{\begin{array}{l}
\frac{d s(t)}{d t}=-\beta(t) i(t) s(t) \\
\frac{d i(t)}{d t}=\beta(t) i(t) s(t)-\gamma(t) i(t), \\
\frac{d r(t)}{d t}=\gamma(t) i(t)
\end{array}\right.
$$

where $s(t)$ is the number of susceptible individuals, $i(t)$ is the number of infected individuals, $r(t)$ is the number of recovered individuals, $\beta(t)$ is the proportional coefficient to the disease transmission rate, and $\gamma(t)$ is the recovery rate (the specific rate at which infected individuals recover from the disease). Let

$N(t)=s(t)+i(t)+r(t)$

the number of individuals in the population, and

$\beta(t)=\frac{\kappa(t) \tau(t)}{N(t)}$,

the proportional coefficient of the disease transmission rate where $\kappa(t)$ is the contact number that an infected individual has per unit of time. $\tau(t)$ is the probability that contacts between an infected and a susceptible individual result in transmission (Martcheva 2015).

The fundamental characteristics of the model (1) parameters are:

C1. the value of the number of contacts that an infected individual has per unit time is greater than zero $(\kappa(t)>$ 0 );

C2. the probability that contact with a susceptible individual result in the transmission is greater than zero $(\tau(t)>0)$; 
C3. the number of individuals in the population is greater than zero $(N(t)>0)$;

C4. the recovery rate is greater than zero $(\gamma(t)>0)$;

To obtain the exact value of $\beta(t)$ is so complex. Several works (Tsay et al. 2020; Köhler et al. 2020; Perkins and España 2020; Kantner and Koprucki 2020; Borri et al. 2020; Péni et al. 2020; Morato et al. 2020a, b) propose new models and new nonlinear estimators to do this. Because it is nontrivial to calculate the $\beta(t)$ value, their controllers become a complex solution.

Here, to keep the proposed controller simple, we use the SIR model (1) considering their parameters, including the population, constants, and a well-mixed population. Then,

$$
\left\{\begin{array}{l}
\frac{d s(t)}{d t}=-\beta_{0} i(t) s(t) \\
\frac{d i(t)}{d t}=\beta_{0} i(t) s(t)-\gamma_{0} i(t) \\
\frac{d r(t)}{d t}=\gamma_{0} i(t)
\end{array}\right.
$$

and

$$
\frac{d N_{0}}{d t}=\frac{d s(t)}{d t}+\frac{d i(t)}{d t}+\frac{d r(t)}{d t}=0,
$$

where the 0 index represents the initial and constant value to the correspondent parameter.

Consider the following assumptions:

A1. the initial condition of $s(t)$ is $s(0)=s_{0}>0$;

A2. the initial condition of $i(t)$ is $i(0)=i_{0}>0$;

Let

$\lim _{t \rightarrow \infty} s(t)=s_{\infty}$,

$\lim _{t \rightarrow \infty} r(t)=r_{\infty}$.

Dividing the derivative of equation $s(t)$ by the derivative of equation $r(t)$, we have

$\frac{d s(t)}{d r(t)}=\frac{-\beta_{0} s(t)}{\gamma_{0}}$

Solving (6), we have

$s(t)=s_{0} \cdot \exp \left(\frac{-\beta_{0} r(t)}{\gamma_{0}}\right)$.

In the worst case $\left(r_{\infty}=N_{0}\right)$, (7) results in

$s(t) \geq s_{0} \cdot \exp \left(\frac{-\beta_{0} N_{0}}{\gamma_{0}}\right)>0$, which implies $s_{\infty}>0$.

The integral form of the $i(t)$, from model (4) is

$i(t)=i_{0} \exp \left(-\gamma_{0} t\right)+\beta_{0} \int_{0}^{t} s(\tau) i(\tau) \exp \left(-\gamma_{0}(t-\tau)\right) d \tau$,

which implies $i(t) \geq 0$, considering the $\mathrm{A} 2$ assumption and (8).

The integral for the first equation of SIR model (4) results in

$$
\begin{aligned}
\int_{0}^{\infty} \dot{s} d t & =-\beta_{0} \int_{0}^{\infty} s(t) i(t) d t \\
s_{\infty}-s_{0} & =-\beta_{0} \int_{0}^{\infty} s(t) i(t) d t
\end{aligned}
$$

Then, considering (8) and (10), we can conclude that

$\lim _{t \rightarrow \infty} i(t)=0$.

Dividing the derivative of the equation $i(t)$ by the derivative of equation $s(t)$, we have

$\frac{d i(t)}{d s(t)}=\frac{\beta_{0} s(t) i(t)-\gamma_{0} i(t)}{-\beta_{0} s(t) i(t)}$.

Integrating (11), we have

$i(t)=-s(t)+\frac{\gamma_{0}}{\beta_{0}} \ln (s(t))+\Xi$,

where $\Xi$ is an arbitrary constant.

In SIR model (4), an epidemic occurs if the number of infected individuals increases, i.e.,

$\frac{d i(t)}{d t}>0$.

Thus, for equation

$\frac{d i(t)}{d t}=\beta_{0} i(t) s(t)-\gamma_{0} i(t)$,

the equilibria are the solutions of equation

$\beta_{0} i(t) s(t)-\gamma_{0} i(t)=0$.

According to the properties of the SIR model, Eq. (15) has two solutions:

1. when $i(t)=0$. This case is referred to as a disease-free equilibrium (the disease is not present in the population);

2 . when $i(t) \neq 0$. This case is referred to as an endemic equilibrium (the disease is present in the population). 
We rewrite Eq. (15) as

$\frac{\beta_{0} s(t)}{\gamma_{0}}=1$

where

$R_{t}=\frac{\beta_{0} s(t)}{\gamma_{0}}$

is the effective reproductive function.

At the outset of an epidemic, nearly everyone (except the index case) is susceptible. So we can say that

$s_{0} \approx N_{0} \gg i_{0}$

Applying (18) in (17), we have the basic reproductive number given by

$R_{0}=\frac{\beta_{0} N_{0}}{\gamma_{0}}=\frac{\kappa_{0} \tau_{0}}{\gamma}$

The $\kappa_{0}$ and $\tau_{0}$ are the constant values for $\kappa(t)$ and $\tau(t)$, respectively.

We say that the disease-free equilibrium is globally stable when $R_{0}<1$, and we say that the endemic equilibrium is globally stable when $R_{0}>1$.

\section{Proposed Controller}

To stop an epidemic outbreak, we can use suppression strategies early on and strong enough. However, these suppression strategies are not a good option for all countries. Some countries, because of the enormous number of vulnerable individuals, can not apply heavy suppression strategies. In these countries, the suppression strategies can produce a catastrophic scenario due to economic factors. On the other hand, defining soft suppression strategies can cause the collapse of the healthcare system. These countries have to manage the epidemic outbreak according to the economics and healthcare effects.

Based on the challenges mentioned above, we wish to design a simple rule to implement, on a local level, a control law to keep the health care system safe with a lower level of social distancing possible. We consider it a simple rule because it does not need specialized facilities to run complex optimization routines. We consider social distancing the number of individuals, infected or not, removed from the population to prevent the spread of infection. We consider "local level" the region around a specific health care system, for example, a city.

\subsection{The Idea}

To a specific epidemic outbreak, consider the peak of infected individuals reaching $15 \%$ of the population. To the 1 million individuals population, the infected individuals get 150 thousand on a specific day and decrease until zero after some days. If we apply the social distancing to 500 thousand individuals of this population, this value goes down to 75 thousand. If we remove from isolation an adequate number of individuals each day when the disease begins to decrease, we can control the epidemic outbreak. This adequate number is proportional to the number of recovered individuals per day. In this way, it is possible to ensure that the number of infected individuals simultaneously never will more than 75 thousand individuals.

According to Kucharski et al. (2020); Li et al. (2020); Prem et al. (2020), in the COVID-19 case, the number of hospitalized individuals is proportional to the number of infected individuals. Considering

$h(t)=H \cdot i(t)$,

the number of hospitalized individuals, where $H>0$ is proportional to the number of infected individuals that need hospitalization, the reduction of hospitalized individuals is proportional to the recovered individuals per day.

Thus, we propose a controller to keep endemic equilibrium when hospitalized individuals achieve the desired number.

The proposed controller has two challenges: the first is the quality of the measurements. The second is the behavior of the spreading disease in individuals getting off the social distancing. The proposed controller action has two characteristics: to avoid the fast-spreading of an epidemic disease, it can keep the individuals isolated; it can not accelerate the recovery of the individuals.

For the first challenge, we propose to use the measurements of hospitalized individuals. This measurement shows a low error and low delay. In this way, the proposed controller, to adjust the social distancing level, uses the difference between the desired setpoint and a measured number of hospitalized individuals.

About the second challenge, it is complex to know the exact behavior of the spreading disease. To avoid this requirement, we will use the endemic equilibrium. When working near the endemic equilibrium, we have an easy way to block a fast-growing of infected individuals that is stop the get-off individuals from social distancing. The number of individuals that get off the isolation is proportional to the number of recovered individuals. In other words, we keep the number of susceptible individuals out of isolation small, and this way, we keep controlled the epidemic outbreak. Even considering huge uncertainties, the epidemic outbreak keeps limited, although it shows some small oscillations. 


\subsection{Acting in the Epidemic Disease}

We use social distancing to act in the epidemic disease. We use it to divide the populations into two groups, and we can change the individuals between the groups. One group is for isolated individuals, and the other group is to free individuals.

Here, we will use social distancing to act in the epidemic disease through the disease incidence, which is the number of individuals who become infected per unit of time. According to SIR model (4), the incidence $(\chi(t))$ is given by

$\chi(t)=\beta_{0} i(t) s(t)=\kappa_{0} \tau_{0} i(t) \frac{s(t)}{N_{0}}$,

in that $s(t) N_{0}^{-1}$ is the probability of contacting a susceptible individual, which results in the accumulated number of infected individuals defined by

$c(t)=\int \chi(t) d t$

When $s(t) \approx N_{0}$, apparently all individuals of the population are susceptible. Considering the basic reproductive number as a constant value, we have the highest incidence value. As the number of susceptible individuals reduces, the incidence value reduces too. Assuming that the restriction measurements will impact $\beta_{0}$, we can represent the effects of the social distancing in the incidence by

$\chi(t)=\rho(t) \kappa_{0} \tau_{0} i(t) \frac{s(t)}{N_{0}}=\rho(t) \beta_{0} i(t) s(t)$

where $\rho(t)$ modulates the mean value of the number of contacts an infected individual has per unit time in normal conditions $\left(\kappa_{0}\right)$. To a well-mixed population, the susceptible population reduction implies reducing the contact number of an individual.

Thus, the SIR model (4) changes to

$$
\left\{\begin{array}{l}
\frac{d s(t)}{d t}=-\rho(t) \beta_{0} i(t) s(t) \\
\frac{d i(t)}{d t}=\rho(t) \beta_{0} i(t) s(t)-\gamma_{0} i(t) . \\
\frac{d r(t)}{d t}=\gamma_{0} i(t)
\end{array}\right.
$$

\subsection{Control Law to Measurements of the $h(t)$}

Consider

$e(t)=h_{d}-h(t)$,

where $h$ indicates the maximum desired infected individuals.

The following assumption is made:
A3. the maximum desired infected individuals is assumed to be a step greater than zero $\left(h_{d}>0\right)$;

The purpose is to find a control law $\rho(t)$ to the system (24), such that the output error $e(t)$ tends to zero when $t \rightarrow \infty$, which guarantees that the health care system is not going to collapse.

Let us consider the control law

$\rho(t)=\frac{\psi(t)}{h(t) s(t) \beta_{0}}$,

where

$\psi(t)=\psi_{1} e(t)+\psi_{2} \int_{0}^{t} e(t) d t$

and $\psi_{1}, \psi_{2}$ are nonnegative constants chosen to adjust the error dynamics. $\psi(t)$ is a PI controller.

Theorem 1 Consider the system of Eq. (24), the error Eq. (25), and the control law (26). Whenever all assumptions $(A 1)-(A 3)$ are satisfied, the error e $(t)$ will converge to zero when time tends to infinity.

Proof Consider the Lyapunov function

$V(\dot{e}, e)=\frac{\dot{e}^{2}}{2}+\frac{\psi_{2} e^{2}}{2 H}$.

Then, the time derivative of $V(\dot{e}, e)$ will be

$\dot{V}(\dot{e}, e)=\dddot{e} \dot{e}+H^{-1} \psi_{2} \dot{e} e$.

By applying (24), (25), and (26) in (29),

$\dot{V}(\dot{e}, e)=-H^{-1} \psi_{1} \dot{e}^{2}-\gamma_{0} \dot{e}^{2} \leq 0$.

For the system to maintain the $\dot{V}(\dot{e}, e)=0$ condition, the trajectory must be confined to the line $\dot{e}=0$. Using the system dynamics (25) yields:

$\dot{e} \equiv 0 \Rightarrow \ddot{e} \equiv 0 \Rightarrow-H^{-1} \psi_{2} e \equiv 0 \Rightarrow e \equiv 0$,

which by LaSalle's theorem, the origin is globally asymptotically stable (GAS).

\subsection{Control Law to Measurements of the $h(t)$ with Uncertainties in the Parameters}

Consider $\hat{\beta}_{0}, \hat{\gamma}_{0}$, and $\hat{H}$ the known values to $\beta_{0}, \gamma_{0}$, and $H$ parameters, respectively, where $\hat{\beta}_{0}=\Delta_{\beta} \beta_{0}, \hat{\gamma}_{0}=\Delta_{\gamma} \gamma_{0}$, and $\hat{H}=\Delta_{H} H$. The $\Delta_{\beta}, \Delta_{\gamma}$, and $\Delta_{H}$ represent the uncertainties in the system. Each of them can assume any value between 0.5 and 1.5. The control law will update to 
$\rho(t)=\frac{\psi(t)}{h(t) s(t) \hat{\beta}_{0}}$.

Corollary 1 Consider the system of Eq. (24), the error Eq. (25), the control law (31), and that there are uncertainties in some of the parameters $\beta_{0}, \gamma_{0}, H$. Whenever all assumptions (A1)-(A3) are satisfied. The error e $(t)$ will converge to zero when time tends to infinity.

Proof Consider the Lyapunov function

$V(\dot{e}, e)=\frac{\dot{e}^{2}}{2}+\frac{\psi_{2} e^{2}}{2 H \Delta_{H} \Delta_{\beta}}$.

Then, the time derivative of $V(\dot{e}, e)$ will be

$\dot{V}(\dot{e}, e)=-\frac{\psi_{1} \dot{e}^{2}}{H \Delta_{H} \Delta_{\beta}}-\gamma_{0} \dot{e}^{2} \leq 0$.

For the system to maintain the $\dot{V}(\dot{e}, e)=0$ condition, the trajectory must be confined to the line $\dot{e}=0$. Using the system dynamics 25 yields:

$\dot{e} \equiv 0 \Rightarrow \ddot{e} \equiv 0 \Rightarrow-\frac{\psi_{2}}{H \Delta_{H} \Delta_{\beta}} e \equiv 0 \Rightarrow e \equiv 0$,

which by LaSalle's theorem, the origin is globally asymptotically stable (GAS).

\subsection{Unmodeled Dynamic Analysis}

Consider $\Delta_{u}(t)$ an unmodeled dynamic in the $s(t)$. Applying $\Delta_{u}(t)$ and the control law (26) into the system (24), the derivative of $i(t)$ change to

$\frac{d i(t)}{d t}=\Delta_{u}(t) \psi(t) H^{-1}-\gamma_{0} i(t)$.

Exist a $\Delta_{d}(t)$ such that

$\Delta_{d}(t)+\psi(t) H^{-1}=\Delta_{u}(t) \psi(t) H^{-1}$.

Thus, we assume $\Delta_{d}(t)$ (unmodeled dynamics in the $s(t)$ ) an input disturbance to the PI controller applied to the first-order plant.

Input disturbance is a known and well-studied problem. In this work, to keep the control law simple, we will ignore it. We can ignore it because of the small number of individuals who get off from isolation per day. With this, we believe that the value of $\Delta_{u}(t) \approx 0$ in this specific situation.

\subsection{Control Law to a Real-Case Epidemic Outbreak}

To a real-case epidemic outbreak without treatment, we update the control law to

$\rho(t)=\max \left(0, \min \left(1, \frac{\psi(t)}{h(t) s(t) \beta_{0}}\right)\right)$.

In other words, $\rho(t)$ can take any values between 0 and 1 . When $h(t)>>h_{d}, \rho(t)<0$, it would be necessary to increase the recovery rate. This situation is possible only with treatment. When $h(t)<<h_{d}, \rho(t)>1$, it would be necessary to increase the contact numbers with infected individuals per unit time. In this situation, we should force new contacts between the individuals. Out of the saturation zones, the control law works as Eq. (26).

Corollary 2 Consider the system of Eq. (24), the error Eq. (25), and the control law (36). Whenever all assumptions (A1)-(A3) are satisfied, and the control law is out of saturated zones, the error e $(t)$ will converge to zero when time tends to infinity.

Proof See Theorem 1.

Theorem 2 Consider the control law (36). Whenever all assumptions (A1)-(A3) are satisfied, system (24) is stable for all time $t>0$.

Proof Based on all assumptions (A1)-(A2), $\beta_{0}>0, \gamma>0$, and $r(t)=N_{0}-s(t)-i(t)$, consider the Lyapunov function

$V(s, i)=s+i$.

Then, the time derivative of $V(s, i)$ will be

$\dot{V}(s, i)=-\gamma i$.

Therefore $\dot{V}(s, i) \leq 0$ implies that the system is stable.

Remark 1 During the period of control law saturation, the value of the integral term of Eq. (27) is not computed.

\section{Numerical Simulations}

In this section, we present and analyze the simulation results of the proposed controller. Some considerations about all simulations are: the numerical method for solving ordinary differential equations was the Euler method; the integration step was one day; they have 365 days; they show the behavior of the infected individuals that will need healthcare; the number of individuals in the population $N_{0}=1$ million; the basic reproductive number $R_{0}=2$ ( $\mathrm{Li}$ et al. 2020; Prem et al. 2020; Kucharski et al. 2020); the recovery rate $\gamma_{0}=0.2(\mathrm{Li}$ 
et al. 2020; Prem et al. 2020; Kucharski et al. 2020); the initial condition of the susceptible individuals $s(0)=N_{0}-1$; the initial condition of the infected individuals $i(0)=1$; the initial condition of the recovered individual $r(0)=0$; and the number of the hospitalized individuals $(h(t))$ is $10 \%$ $(H=0.1)$ of the infected individuals.

The uncertainties considered in the values of $H, \beta_{0}, \gamma$ were $-5 \%,+15 \%$, and $-20 \%$, respectively. The unmodeled dynamic is

$\Delta_{u}(t)=1+0.02 \sin \left(\frac{2 \pi}{7} t\right)+0.015 \varphi$,

where $\varphi \sim \mathcal{N}(0,1)$ normally distributed with mean 0 and standard deviation 1.

The value $h_{d}=2500$ is equivalent to 2.5 Hospital beds per 1000 population. Value equals to the United Kingdom in 2019 according to OECD - Organization for Economic Co-Operation and Development.

\subsection{Proposed Controller}

All simulations in this subsection (Figs. 1, 2, 3, 4) uses the model (24), the control law is (36), and the gains of the controller are $\psi_{1}=0.01, \psi_{2}=0.015$. The simulations of Fig. 1 uses $\rho(t)=1$ all the time.

The simulation of Fig. 1 does not use the proposed controller and applies three different values to $R_{0}$. We can see, in Fig. 1, the effects of an epidemic disease without a controller. It is possible to see that the hospitalized individuals show a peak. During the peak, the health care system does not support the number of hospitalized individuals. Another aspect is that the accumulated number of infected individuals is less when $R_{0}$ goes down.

The simulation of Fig. 2 uses the proposed controller to adjust the social distancing level with and without uncertainties in the parameters. In this simulation, it is possible to see that the number of hospitalized individuals $(h(t))$ goes to the desired value $\left(h_{d}\right)$. However, to do this, we need to impose a social distancing during some days. The number of days is inversely proportional to the desired number of hospitalized individuals. In the beginning, social distancing is near $45 \%$ and shows a soft reduction during 100 days.

The simulation of Fig. 3 uses the proposed controller to adjust the social distancing level without uncertainties in the parameters to different $R_{0}$ scenarios. We simulate three $R_{0}$ values, 2.5, 2.0, and 1.5. In this simulation, it is possible to see that the social distancing level depends on the basic reproductive number and the proportion of susceptible individuals in the population, which is according to the mathematical properties of the SIR model, Eqs. (6)-(19). Thus, considering the same population conditions, the peak of social distancing level is an exponential function of $R_{0}$. Similar to the social distancing level, the number of days that need any restriction measure is an exponential function of $R_{0}$. These characteristics are independent of the control technique used.

The simulation of Fig. 4 uses the proposed controller to adjust the social distancing level with uncertainties in the parameters and unmodeled dynamics (39). In this simulation, it is possible to see that the number of hospitalized individuals $(h(t))$ keeps inside the desired number of hospitalized individuals $\left(h_{d}\right)$ range. The unmodeled dynamics have seven days period with an oscillation of $7 \%$ of the number of susceptible individuals. This result demonstrates that the proposed controller is robust to the challenges of the real epidemic outbreak.

\subsection{Proposed Controller Using Steps to Reopen}

All simulations in this subsection (Figs. 5, 6, 7) uses the model (24), the control law is (36), and the gains of the controller are $\psi_{1}=0.01, \psi_{2}=0.015$. The social distancing will change only when $\rho(t)$ falls or grows $5 \%$ of the total population.

One of the challenges to use the proposed controller is the soft value to reopen. It can be very complex for decisionmakers to do this. To overcome this challenge, we propose to reopen by steps of $5 \%$ of the total population. We see in Fig. 5 the value of hospitalized individuals oscillating inside a predefined range. These oscillations occur because of the nonlinearities of the system. We also can see that the duration of the highest social distancing level is of approximately 20 days, and after 100 days, it is not more necessary social distancing.

The simulation of Fig. 6 uses the proposed controller using steps to reopen to adjust the social distancing level with uncertainties in the parameters and unmodeled dynamics (39). We see, again, the proposed controller keeping the number of the hospitalized individuals oscillating inside a predefined range. It is a more realistic scenario because of considering uncertainties and unmodeled dynamics.

In the simulation of Fig. 7, we change the desired number of hospitalized individuals from $h_{d}=2500$ to $h_{d}=1250$. We do this because 2.5 Hospital beds per 1000 population is a high value to rich countries and maybe impossible value to economically vulnerable countries. When we reduce the Hospital beds available at the half, the days with some social distance level approximately doubles. Though the time doubles, the proposed controller keeps the number of hospitalized individuals oscillating inside a predefined range.

\subsection{Compiling the Results}

We compile all simulations of Figs. 2, 3, 4, 5, 6, 7 into Fig. 8. The objective is to highlight the behavior in all scenarios together. The blue region has the upper and lower values for 


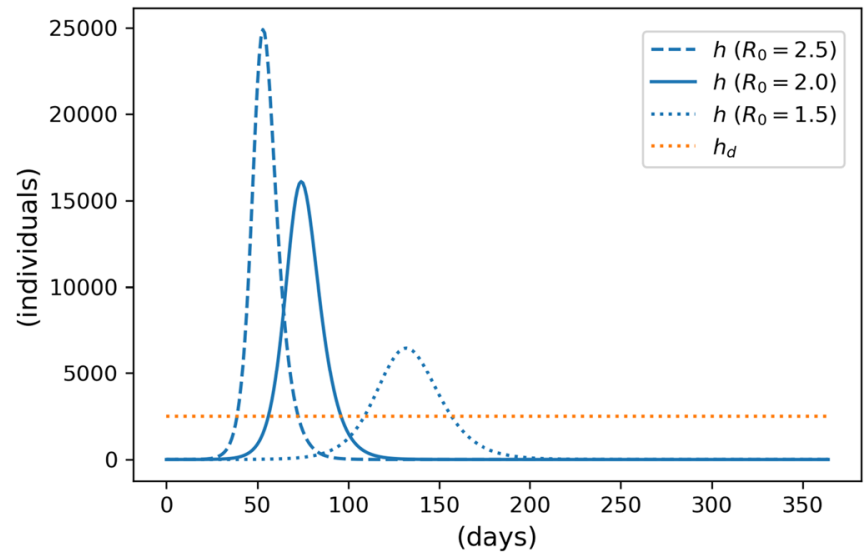

Fig. 1 Simulation of SIR model without a controller, where the blue line, dashed blue line, and dotted blue line are the number of the hospitalized individuals $h$. The orange dotted line is the desired number

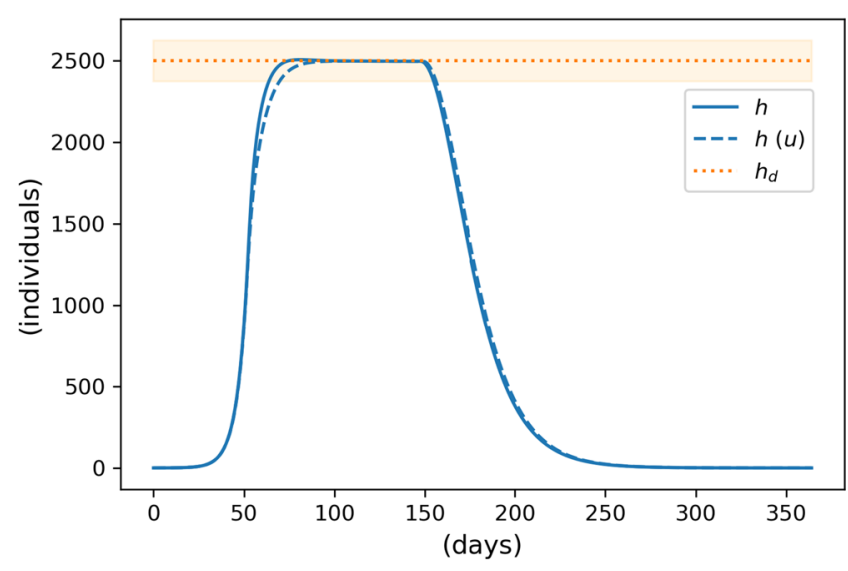

Fig. 2 Simulation of the SIR model with the proposed controller, where the blue line is the number of the hospitalized individuals, the dashed blue line is the number of the hospitalized individuals with uncertainties in the parameters. The orange dotted line is the desired hospitalized

$h(t)$ of all simulations. In Fig. 8, we also see a $16 \%$ reduction in the number of accumulated cases comparing the cases with and without the proposed controller.

We compile all simulations to the same set of cases of Figs. 2, 3, 4, 5, 6, 7, when uses $h_{d}=1250$, in Fig. 9. In this figure, we see the number of hospitalized individuals inside a predefined range. We also see a $20 \%$ reduction in the number of accumulated cases comparing the cases with and without the proposed controller.

\subsection{The Proposed Controller Considering Delays}

For many diseases, the infected individuals do not become immediately infectious. The pathogen needs time to replicate and establish itself in the new host. The time during which an individual is infected but not yet infectious is called the latent

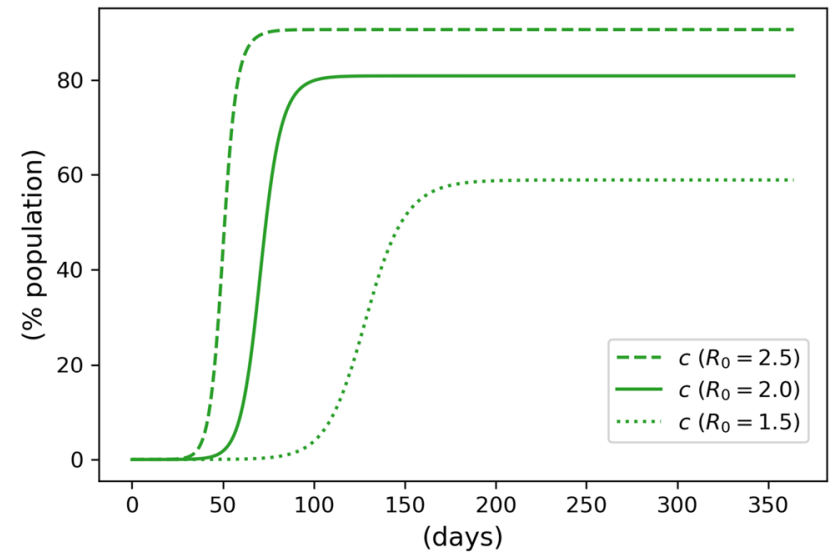

of hospitalized individuals $h_{d}$. The accumulated numbers of infected individuals $(c)$ are the green line, dashed green line, and dotted green line (Color figure online)

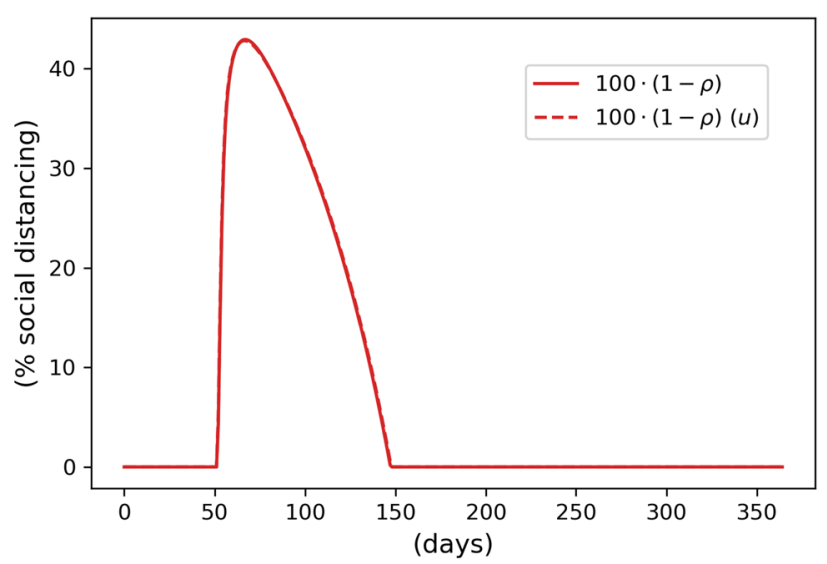

individual's number. The light orange region indicates the range of $\pm 5 \%$ of the $h_{d}$, the red line is the percent of the social distancing required, and the dashed red line is the percent of the social distancing required when there are uncertainties in the parameters (Color figure online)

period (Martcheva 2015). We use the latent period because it is more realistic for our scenario. We adopt, as feedback, the measurement of the hospitalized individuals, which shows an insignificant dead-time.

Now, introducing a latent period in the SIR model (24), we obtain

$\left\{\begin{array}{l}\frac{d s(t)}{d t}=-\rho(t) \beta_{0} i(t) s(t) \\ \frac{d x(t)}{d t}=\rho(t) \beta_{0} i(t) s(t)-\gamma_{0} i(t) \\ \frac{d i(t)}{d t}=\eta_{0} x(t)-\gamma_{0} i(t) \\ \frac{d r(t)}{d t}=\gamma_{0} i(t)\end{array}\right.$,

where $\left(\eta_{0}\right)^{-1}$ is the latent period. Equation (40) is the SEIR model. 


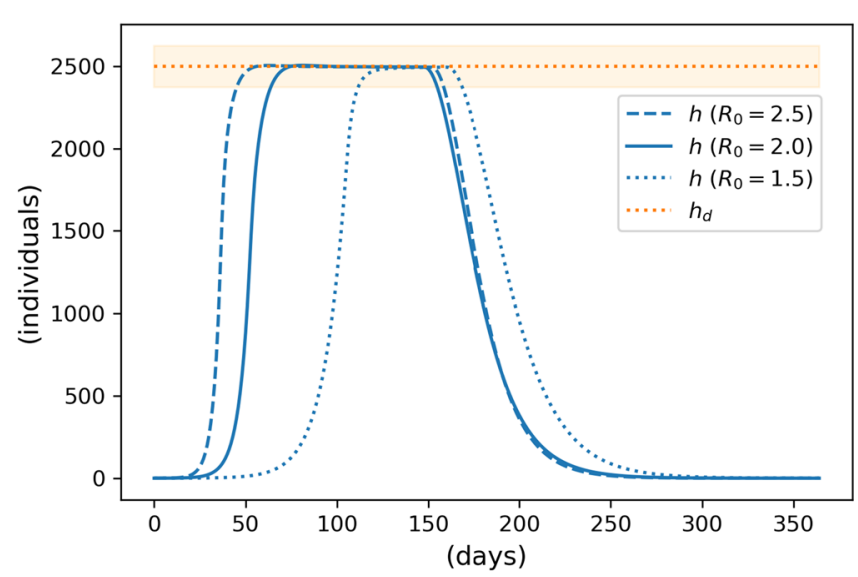

Fig. 3 Simulation of SIR model with the proposed controller, where the blue line, dashed blue line, and dotted blue line are the number of the hospitalized individuals to $R_{0}=2.0, R_{0}=2.5$, and $R_{0}=1.5$, respectively. Orange dotted line is the desired hospitalized individu-

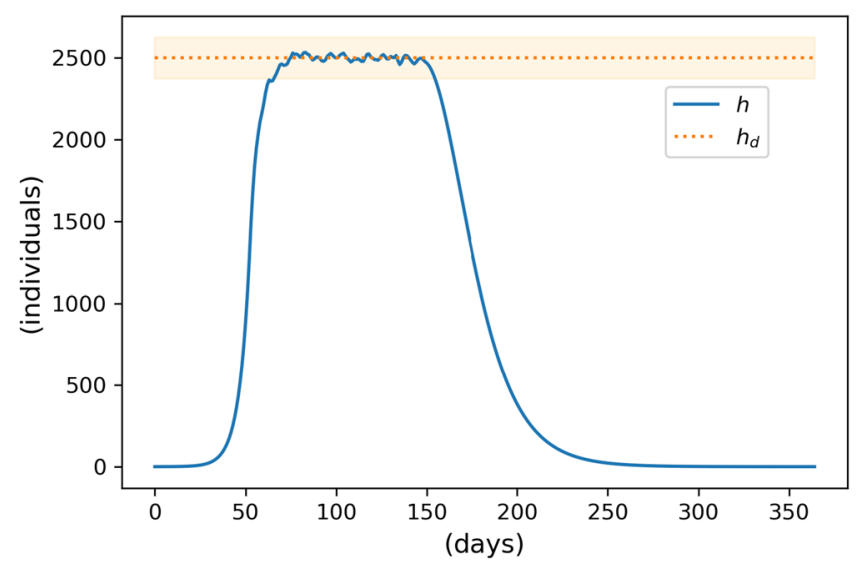

Fig. 4 Simulation of SIR model with the proposed controller, where the blue line is the number of the hospitalized individuals with uncertainties in the parameters and unmodeled dynamics, orange dotted line is the number of desired hospitalized individuals. The light orange region

All simulations in this subsection (Figs. 10,11) uses the model (40), the control law is (36), the latent period is ten days $\left(\eta_{0}=0.1\right)$, and the gains of the controller are $\psi_{1}=0.22$, $\psi_{2}=0.025$. The simulations of Fig. 10 uses $\rho(t)=1$ all the time.

The simulation of Fig. 10 does not use the proposed controller and applies three different values to $R_{0}$. We can see, in Fig. 10, the effects of an epidemic disease with latent period and without a control law. It is possible to see a peak of hospitalized individuals slower and lower than the SIR model in open-loop mode (Fig. 1). During the peak, the health care system does not support the number of hospitalized individuals. Another aspect is that the accumulated number of infected individuals is less when $R_{0}$ goes down.

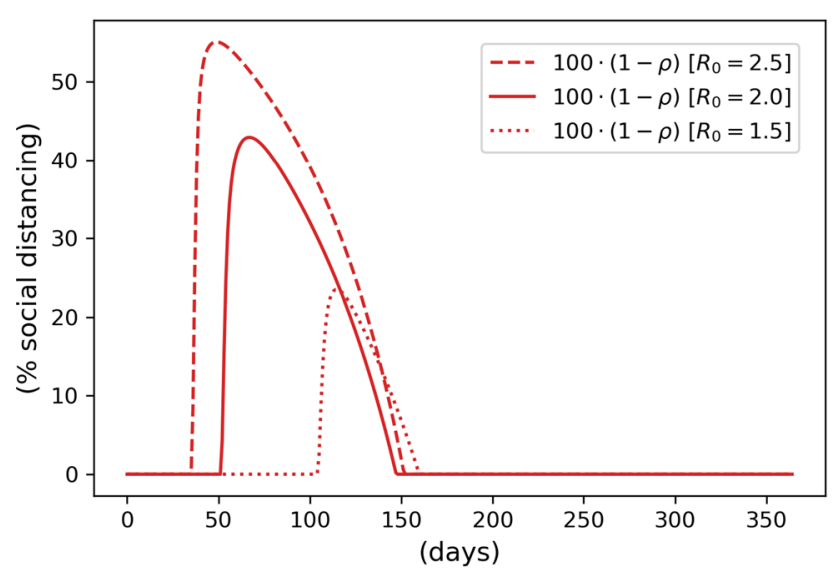

als'. The light orange region indicates the range of $\pm 5 \%$ of the $h_{d}$, the red line is the percent of the social distancing required, and the dashed red line is the percent of the social distancing required when there are uncertainties in the parameters (Color figure online)

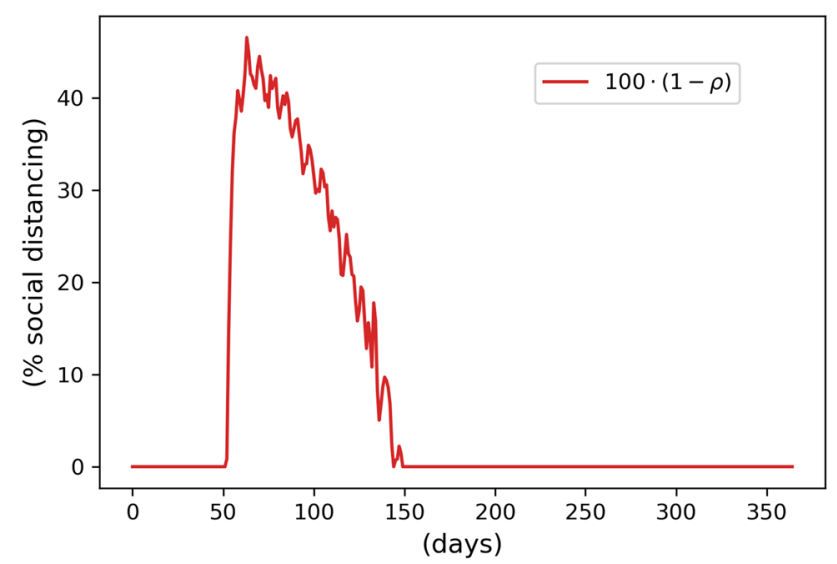

indicates the range of $\pm 5 \%$ of the $h \_d$, and the red line is the percent of the social distancing required when there are uncertainties in the parameters and unmodeled dynamics (Color figure online)

The simulation of Fig. 11 uses the proposed controller to adjust the social distancing level. In this simulation, it is possible to see that the number of hospitalized individuals $(h(t))$ keeps lower than the desired number of hospitalized individuals $\left(h_{d}\right)$. However, to do this, we need to impose a social distancing during some days. The number of days is inversely proportional to the desired number of hospitalized individuals. In the beginning, social distancing grows up soft until near 35\% and shows a soft reduction until the finish. The duration of social distancing is approximately 100 days.

The small oscillation's peak at the beginning of the social distance in Fig. 11 is due to anti-reset wind-up (Remark 1). We do not solve this to do not change the proposed control law. 


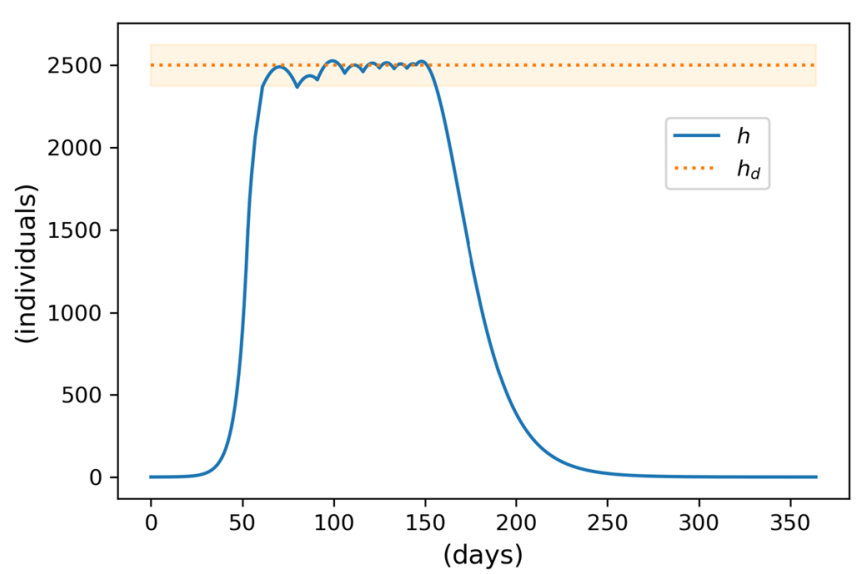

Fig. 5 Simulation of SIR model with the proposed controller, where the blue line is the number of the hospitalized individuals, the orange dotted line is the number of desired hospitalized individuals. The light

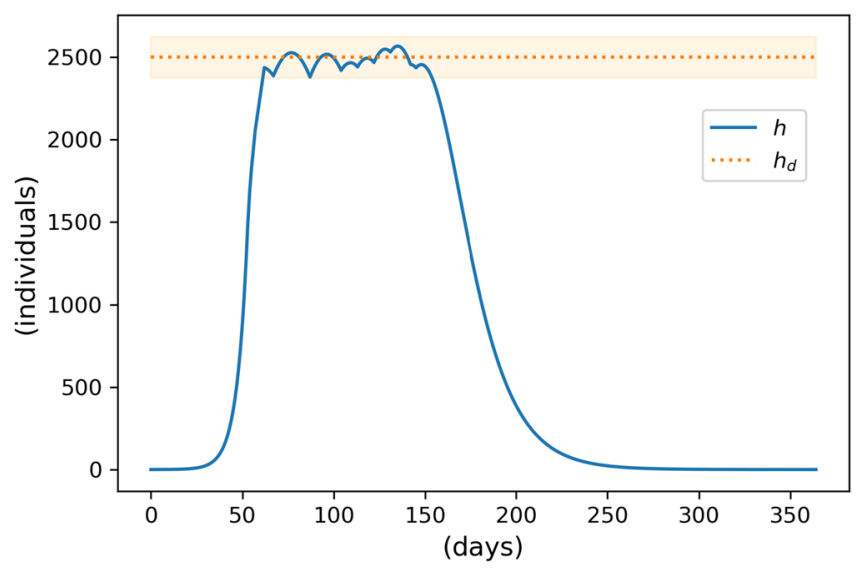

Fig. 6 Simulation of SIR model with the proposed controller, where the blue line is the number of the hospitalized individuals with uncertainties in the parameters and unmodeled dynamics, orange dotted line is the number of desired hospitalized individuals. The light orange region

\subsection{Discussion}

In this work, we propose a closed-loop controller to control the spreading of an epidemic disease. The proposed controller uses only measurements of the number of hospitalized individuals and acts just on the social distancing level.

Try to use an open-loop control to adjust the level of social distancing could be tragic. In this situation, the number of hospitalized individuals can keep upper than the desired value. Also, the number of days with some social distancing level can be greater than necessary. Other implications of the open-loop approach are: the accumulated number of infected individuals can keep above the value of the closedloop case; the number of hospitalized individuals can show huge oscillations due to the nonlinear characteristic of an epidemic disease.

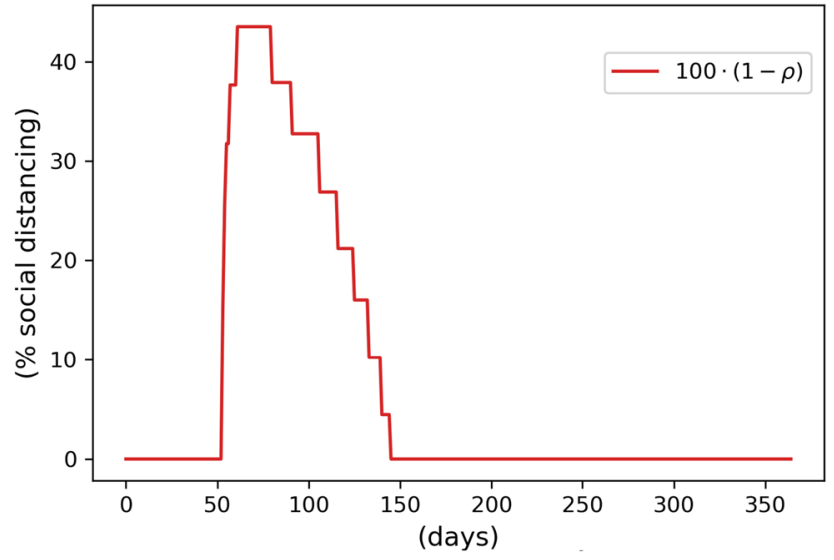

orange region indicates the range of $\pm 5 \%$ of the $h \_d$, and the red line is the percent of the social distancing required (Color figure online)

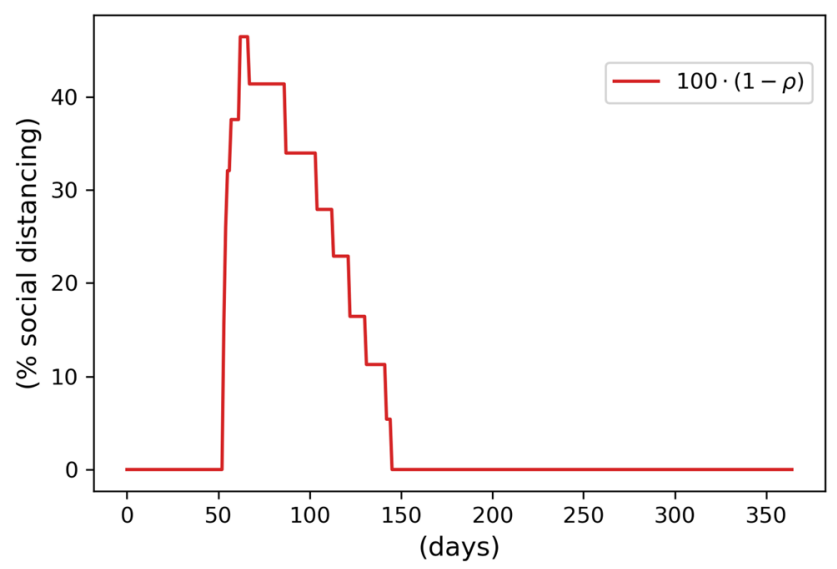

indicates the range of $\pm 5 \%$ of the $h \_d$, and the red line is the percent of the social distancing required when there are uncertainties in the parameters and unmodeled dynamics (Color figure online)

The $R_{0}$ Effects The basic reproductive number $R_{0}$ can change the behavior of an epidemic disease. If the $R_{0}$ grows, the duration of epidemic disease shortens, and the peak of the hospitalized individuals and the number of accumulated infected individuals increases.

Feedback Signal To measure the behavior of the epidemic outbreak, we use the number of hospitalized individuals. We choose this variable because it shows a lower error and delay. It also does not need massive tests of the population.

Control Signal To act about the epidemic outbreak, we use social distancing. We apply social distancing through quarantine to ensure that individuals, infected or not, will be removed from the population. About steps to reopen: 5\% is an example. We can use other values to the steps. The size of the step is according to the limitations of the pop- 


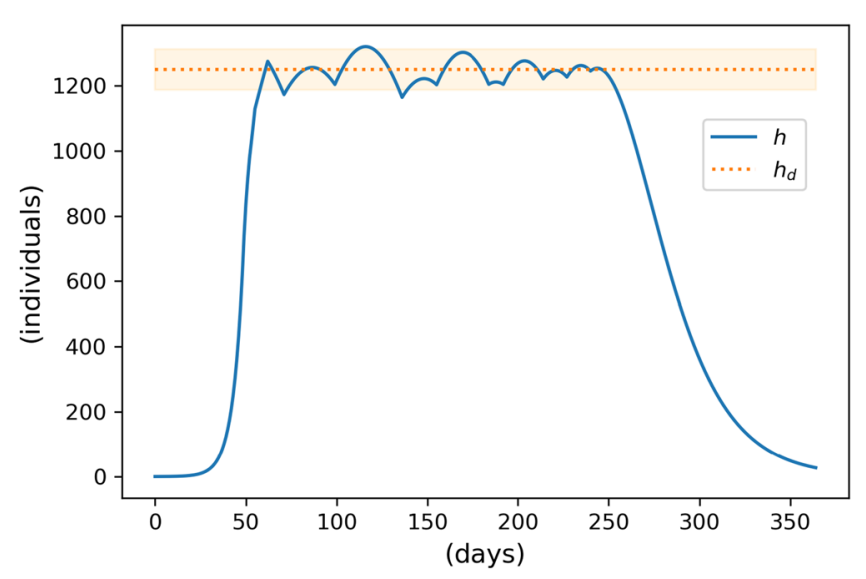

Fig. 7 Simulation of SIR model with the proposed controller, where the blue line is the number of the hospitalized individuals with uncertainties in the parameters and unmodeled dynamics, orange dotted line is the number of desired hospitalized individuals. The light orange region

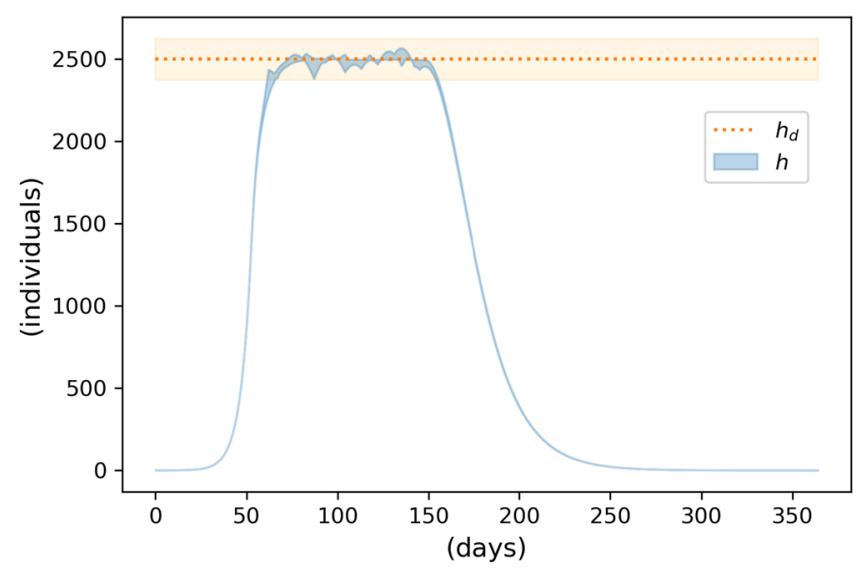

Fig. 8 Compiled result of simulations of Figs. 2, 3, 4, 5, 6, 7, where the blue fill represents the region made of upper and lower values for $h(t)$. Orange dotted line is the number of desired hospitalized individuals. The light orange region indicates the range of $5 \%$ of the $h \_d$. The

ulation. However, the reader can notice that in a 1 million population, 5\% represent, for example, a neighborhood or all professionals in the specific segment. Then, the decisionmakers will define: professors stay home, this neighborhood stay home, etc. The decision-makers indeed will need to be more organized, but this is not a big challenge. The vaccination process requires a more refined control signal than we propose. It works with a lower percentage of the population.

Social Distancing Level To be more didactic, we chose a range from $0 \%$ to $100 \%$ to the level of social distancing. $0 \%$ means that no one measure is adopted, and $100 \%$ means the maximum social distancing level is required. We consider personal protective measures, including hand hygiene, respiratory etiquette, face masks, and environmental measures,

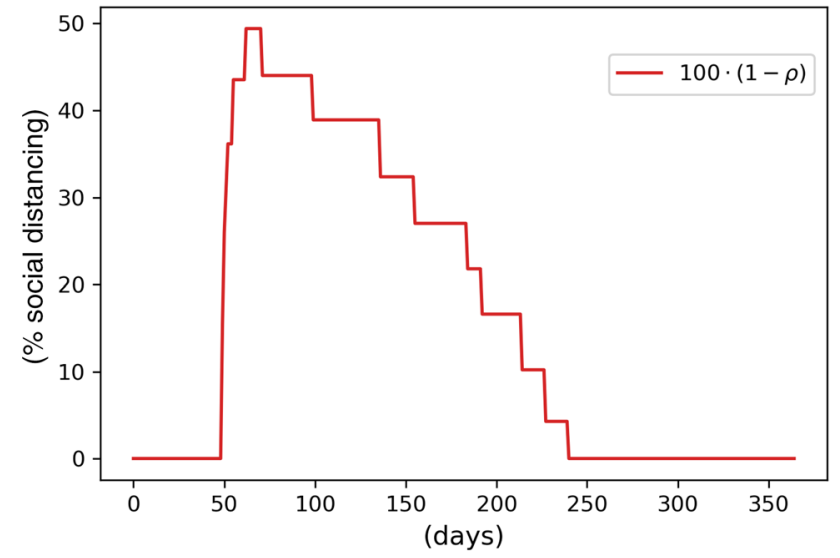

indicates the range of $\pm 5 \%$ of the $h \_d$, and the red line is the percent of the social distancing required when there are uncertainties in the parameters and unmodeled dynamics (Color figure online)

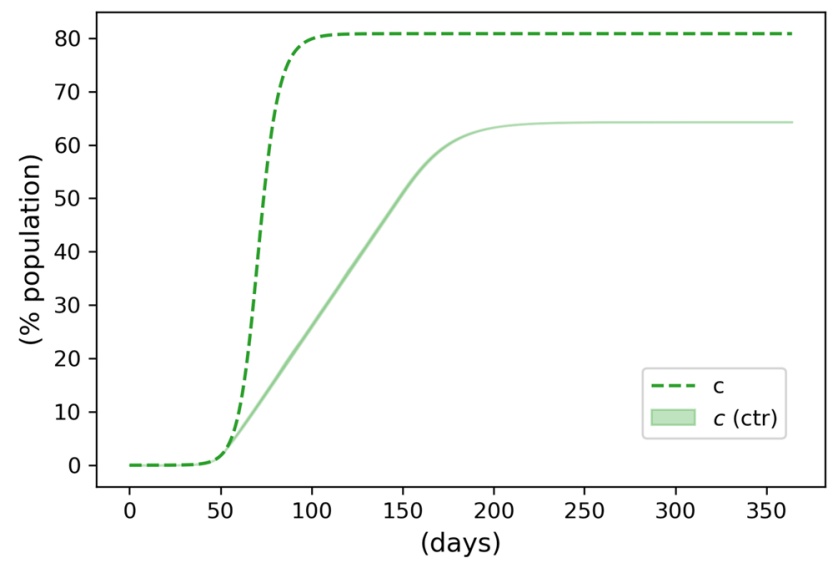

dashed green line is the accumulated number of infected individuals $c(t)$ without a proposed controller. The light green region is the upper and lower accumulated number of infected individuals when using the proposed controller (Color figure online)

as factors that can influence the social distancing level. In other words, $0 \%$ means none measure, including no personal protective measures.

Adjustment of the Controller Parameters The designer can use the following logic to calculate the value of each gain of the proposed controller: $\psi_{1}$ is related to how fast the error $(e(t))$ goes to nearby zero, and $\psi_{2}$ is related to how softly the error $(e(t))$ converges to zero. All simulations of the proposed controller, except the simulation with a latent period, use the same gains. We did this to demonstrate that the gains adjustment is not so sensitive.

Model Dependency The proposed control law uses the endemic equilibrium. This strategy is less model-depen- 


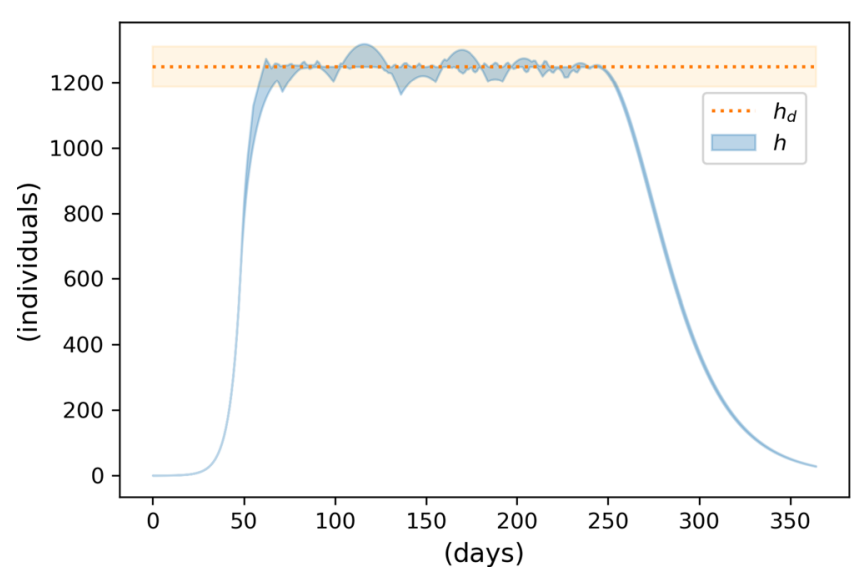

Fig. 9 Compiled result of simulations to the same set of cases of Figs. 2, $3,4,5,6,7$ when uses $h_{d}=1250$, where the blue fill represents the region made of upper and lower values for $h(t)$. Orange dotted line is the number of desired hospitalized individuals. The light orange region indicates the range of $\pm 5 \%$ of the $h \_d$. The dashed green line is the

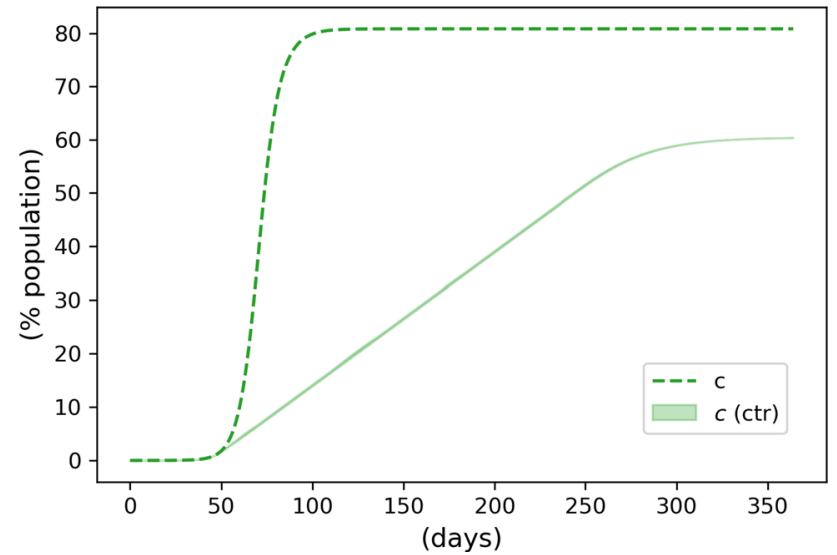

accumulated number of infected individuals $c(t)$ without a proposed controller. The light green region is the upper and lower accumulated number of infected individuals when using the proposed controller (Color figure online)
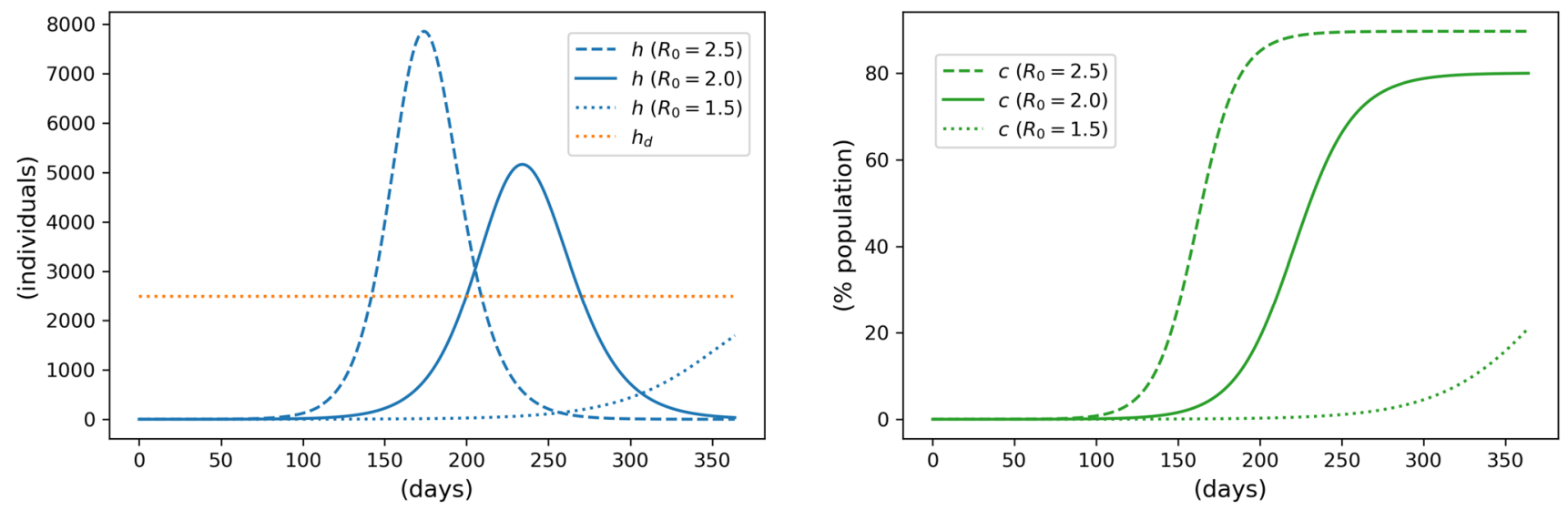

Fig. 10 Simulation of SIR model without a controller, where the blue line is the number of the hospitalized individuals $h$, orange dotted line is the desired number of hospitalized individuals $h_{d}$, and the green line is the accumulated number of infected individuals $c$ (Color figure online)

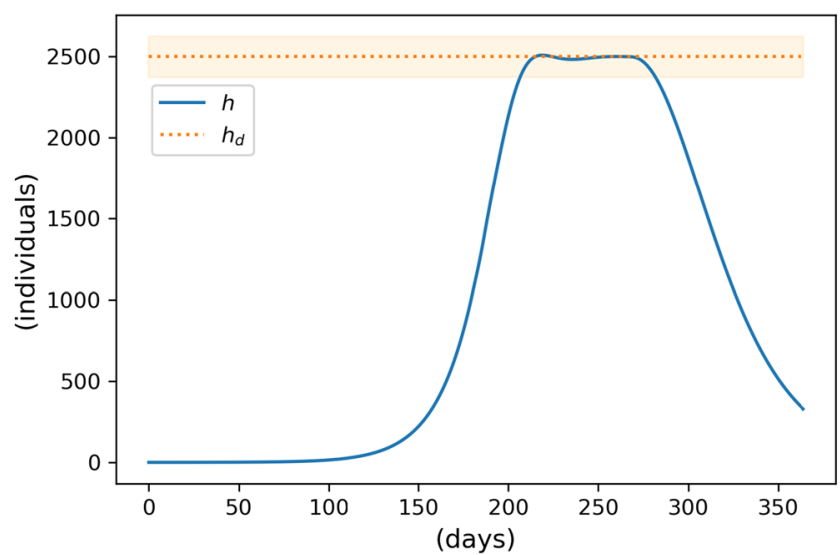

Fig. 11 Simulation of SIR model with the proposed controller, where the blue line is the number of the hospitalized individuals, orange dotted line is the desired hospitalized individuals number. The light orange

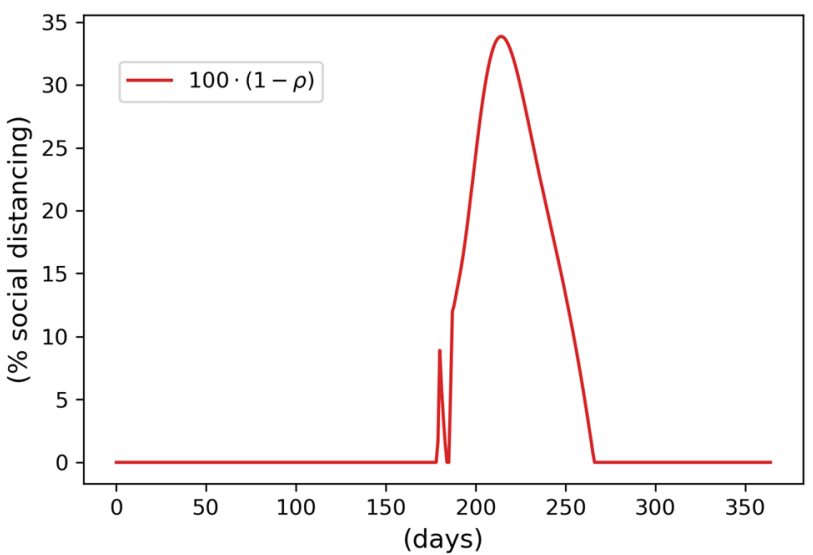

region indicates the range of $\pm 5 \%$ of the $h_{d}$, and the red line is the percent of the social distancing required (Color figure online) 
dent and does not need the exact value of model parameters (see Corollary 1). The uncertainties in the parameters produce their most influence on the initial overshoot. When the epidemic disease is in endemic equilibrium, the system's behavior shows linear characteristics, and we can use a simple control law to keep it in the equilibrium.

Propose Controller to Real Epidemic Disease The proposed controller is simple, only needs measurements of hospitalized individuals' numbers, and uses a daily update scheme. We consider it a viable option because it does not require massive swab tests and highly qualified teams to manage it. Moreover, it can use steps to reopen, which can facilitate its application by the decision-makers.

Performance The proposed controller to $h_{d}=2500$ requires 100 days of social distancing and to $h_{d}=1250$ requires 200 days of social distancing. For the first case, after 60 days, the social distancing is below $30 \%$. For the second case, after 100 days, the social distancing is below $30 \%$. In steps-to-reopen schemes, the controller never regresses in the reopen. The highest social distancing is lower than $50 \%$ and by a couple of days. In all cases, the proposed controller ensures the number of hospitalized individuals below the upper limit of a predefined range. The proposed controller achieves its objective until when there is a latent period.

The Social-Economic Cost The proposed controller does not impose, any moment, a high level of socials distancing. This characteristic allows the region to keep some economic activity and protect the most vulnerable persons. We believe that controlling endemic equilibrium is the safe and fast way to overcome an epidemic outbreak.

Proposed Control Versus On-off Controllers We decide to use endemic equilibrium and keep the number of hospitalized individuals inside a predefined range. We did this to develop a more simple controller when compared to on-off strategies. The proposed controller imposes a high level of social distancing when the number of infected individuals is low. In other words, approximately all populations removed are susceptible. When we put back these individuals, we do this in small quantities of susceptible individuals and when the system is in the endemic equilibrium. This strategy reduces the nonlinear effects of the system. The on-off controllers impose a high level of social distancing by rounds. To each round, we remove from the population more individuals in the three classes. The onoff controllers are always working in a nonlinear zone and with a heterogeneous population, which results in complex strategies.
Local-Level Control Law To develop a simple control law, we assume that the population is sufficiently well mixed. Thus, instead of applying the proposed controller to a country, we recommend using the proposed controller to a region around a specific health care system, for example, a city. In this way, each region will control its epidemic outbreak. The spreading of an epidemic disease is not homogeneous, and each region of a country can be affected in different moments with different intensities. To use a global control action in this scenario will impose inadequate restrictions for long periods. To apply effective local actions to control the epidemic outbreak has several successful cases in the literature (Martcheva 2015; Daley and Gani 1999). The most recent successful case was Wuhan in China.

\section{Conclusions}

This work proposed a simple control law with low model dependency based on the endemic equilibrium to epidemic outbreaks. The proposed controller calculates the social distancing level to keep the epidemic outbreak controlled. The social distancing level is adjusted to guarantee the fastest way to finish the outbreak with the number of hospitalized individuals below the desired value. This technique can reduce the economic problems of social distancing and keeps the health care system working.

We simulate the proposed controller applied to the ideal case, considering uncertainties, unmodeled dynamics, a ten days latent period, and different values of the desired number of hospitalized individuals. In all cases, the proposed controller ensures the number of hospitalized individuals lower than the upper limit of a predefined range. This result demonstrates the robustness of the proposed controller.

The proposed controller has two main characteristics: it is simple and can operate using steps to reopen; it keeps the number of hospitalized individuals approximately constant during all the time of the epidemic outbreak. These characteristics are essential to allow better management of the resources to suppress the epidemic disease. They also help the decision-makers adopt the proposed controller. The first characteristic simplifies the reopening process because it uses, like a step, a determined percentage of the population. The second characteristic is fundamental to help in the healthcare system planning.

For all cases, the highest social distancing is lower than $50 \%$ and by a couple of days. This result is relevant because it keeps some economic activity and protects the most vulnerable persons in developing countries.

To better results, we have to apply the proposed controller early on. The proposed controller reduces the number of accumulated infected individuals, and because of low model dependency does not need a "careful estimator". 
In the future, we will expand the proposed controller to a group-structure SIR model and apply it to huge regions, for example, a country. A group-structure SIR model is considered adequate for epidemic outbreaks in heterogeneous populations.

Acknowledgements An early version of paper was presented at XXIII Congresso Brasileiro de Automática (CBA 2020).

\section{References}

Allen, L. J. (2017). A primer on stochastic epidemic models: Formulation, numerical simulation, and analysis. Infectious Disease Modelling, 2(2), 128-142. https://doi.org/10.1016/j.idm.2017.03. 001.

Ball, F. G., Knock, E. S., \& O’Neill, P. D. (2008). Control of emerging infectious diseases using responsive imperfect vaccination and isolation. Mathematical Biosciences, 216(1), 100-113. https://doi. org/10.1016/j.mbs.2008.08.008.

Borase, R. P., Maghade, D. K., Sondkar, S. Y., \& Pawar, S. N. (2021). A review of pid control, tuning methods and applications. International Journal of Dynamics and Control, 9(2), 818-827. https:// doi.org/10.1007/s40435-020-00665-4.

Borri, A., Palumbo, P., Papa, F., \& Possieri, C. (2020). Optimal design of lock-down and reopening policies for early-stage epidemics through sir-d models. Annual Reviews in Control,. https://doi.org/ 10.1016/j.arcontrol.2020.12.002.

Casella, F. (2021). Can the covid-19 epidemic be controlled on the basis of daily test reports? IEEE Control Systems Letters, 5(3), 10791084. https://doi.org/10.1109/LCSYS.2020.3009912.

Daley, D. J., \& Gani, J. (1999). Epidemic modelling: An introduction. Cambridge Studies in Mathematical Biology, Cambridge University Press,. https://doi.org/10.1017/CBO9780511608834.

Di Lauro, F., Kiss, I. Z., Rus, D., \& Della Santina, C. (2021). Covid19 and flattening the curve: A feedback control perspective. IEEE Control Systems Letters, 5(4), 1435-1440. https://doi.org/ 10.1109/LCSYS.2020.3039322.

Gaff, H., \& Schaefer, E. (2009). Optimal control applied to vaccination and treatment strategies for various epidemiological models. Mathematical Biosciences \& Engineering,. https://doi.org/10.3934/ mbe.2009.6.469.

Hellewell, J., Abbott, S., Gimma, A., et al. (2020). Feasibility of controlling covid-19 outbreaks by isolation of cases and contacts. The Lancet Global Health, 8(4), e488-e496. https://doi.org/10.1016/ S2214-109X(20)30074-7.

Isidori, A. (1995). Nonlinear Control Systems. Communications and Control Engineering. London: Springer.

Jiang, J., \& Zhou, T. (2018). Resource control of epidemic spreading through a multilayer network. Scientific Reports, 8(1), 1629. https://doi.org/10.1038/s41598-018-20105-w.

Kantner, M., \& Koprucki, T. (2020). Beyond just "flattening the curve": Optimal control of epidemics with purely non-pharmaceutical interventions. Journal of Mathematics in Industry,10(1), 23. https://doi.org/10.1186/s13362-020-00091-3.

Kermack, W. O., McKendrick, A. G., \& Walker, G. T. (1927). A contribution to the mathematical theory of epidemics. Proceedings of the Royal Society of London Series A, Containing Papers of a Mathematical and Physical Character, 115(772), 700-721. https://doi. org/10.1098/rspa.1927.0118.

Köhler, J., Schwenkel, L., Koch, A., Berberich, J., Pauli, P., \& Allgöwer, F. (2020). Robust and optimal predictive control of the covid-19 outbreak. Annual Reviews in Control,. https://doi.org/10.1016/j. arcontrol.2020.11.002.
Kucharski, A. J., Russell, T. W., Diamond, C., et al. (2020). Early dynamics of transmission and control of covid-19: A mathematical modelling study. The Lancet Infectious Diseases, 20(5), 553-558. https://doi.org/10.1016/S1473-3099(20)30144-4.

Li, Q., Guan, X., Wu, P., et al. (2020). Early transmission dynamics in Wuhan, China, of novel coronavirus-infected pneumonia. New England Journal of Medicine, 382(13), 1199-1207. https://doi. org/10.1056/NEJMoa2001316.

Martcheva, M. (2015). An Introduction to Mathematical Epidemiology. Texts in Applied Mathematics. Berlin: Springer.

Morato, M. M., Bastos, S. B., Cajueiro, D. O. \& Normey-Rico, J. E. (2020a). An optimal predictive control strategy for covid-19 (sars-cov-2) social distancing policies in brazil. Annual Reviews in Control 50:417-431. https://doi.org/10.1016/j.arcontrol.2020. 07.001 .

Morato, M. M., Pataro, I. M., Americano da Costa, M. V., \& NormeyRico, J. E. (2020b). A parametrized nonlinear predictive control strategy for relaxing covid-19 social distancing measures in brazil. ISA Transactions,. https://doi.org/10.1016/j.isatra.2020.12.012.

Newman, M. E. J. (2002). Spread of epidemic disease on networks. Physical Review E,66, 016128. https://doi.org/10.1103/ PhysRevE.66.016128.

Nowzari, C., Preciado, V. M., \& Pappas, G. J. (2016). Analysis and control of epidemics: A survey of spreading processes on complex networks. IEEE Control Systems Magazine, 36(1), 26-46.

Péni, T., Csutak, B., Szederkényi, G., \& Röst, G. (2020). Nonlinear model predictive control with logic constraints for covid-19 management. Nonlinear Dynamics, 102(4), 1965-1986. https://doi. org/10.1007/s11071-020-05980-1.

Perkins, T. A., \& España, G. (2020). Optimal control of the covid19 pandemic with non-pharmaceutical interventions. Bulletin of Mathematical Biology, 82(9), 118. https://doi.org/10.1007/ s11538-020-00795-y.

Prem, K., Liu, Y., Russell, T. W., et al. (2020). The effect of control strategies to reduce social mixing on outcomes of the covid-19 epidemic in Wuhan, China: a modelling study. The Lancet Public Health. https://doi.org/10.1016/S2468-2667(20)30073-6.

Roberts, M., Andreasen, V., Lloyd, A., \& Pellis, L. (2015). Nine challenges for deterministic epidemic models. Epidemics, 10, 49-53.

Sélley, F., Besenyei, Á., Kiss, I. Z., \& Simon, P. L. (2015). Dynamic control of modern, network-based epidemic models. SIAM Journal on Applied Dynamical Systems, 14(1), 168-187. https://doi.org/ $10.1137 / 130947039$.

Stewart, G., Heusden, K., \& Dumont, G. A. (2020). How control theory can help us control covid-19. IEEE Spectrum, 57(6), 22-29. https://doi.org/10.1109/MSPEC.2020.9099929.

Tsay, C., Lejarza, F., Stadtherr, M. A., \& Baldea, M. (2020). Modeling, state estimation, and optimal control for the us covid-19 outbreak. Scientific Reports, 10(1), 10711. https://doi.org/10.1038/s41598020-67459-8.

Verity, R., Okell, L. C., Dorigatti, I., et al. (2020). Estimates of the severity of coronavirus disease 2019: a model-based analysis. The Lancet Infectious Diseases. https://doi.org/10.1016/S14733099(20)30243-7.

Zaman, G., Kang, Y. H., \& Jung, I. H. (2009). Optimal treatment of an sir epidemic model with time delay. Biosystems, 98(1), 43-50. https://doi.org/10.1016/j.biosystems.2009.05.006.

Zhang, Z., Wang, H., Wang, C., \& Fang, H. (2015). Modeling epidemics spreading on social contact networks. IEEE Transactions on Emerging Topics in Computing, 3(3), 410-419. https://doi.org/ 10.1109/TETC.2015.2398353.

Publisher's Note Springer Nature remains neutral with regard to jurisdictional claims in published maps and institutional affiliations. 